University of Wollongong

Research Online

Faculty of Engineering and Information

Faculty of Engineering and Information

Sciences - Papers: Part A

Sciences

$1-1-2016$

Reproducing personal sound zones using a hybrid synthesis of dynamic and parametric loudspeakers

Jacob Donley

University of Wollongong, jrd089@uowmail.edu.au

Christian H. Ritz

University of Wollongong, critz@uow.edu.au

Bastiaan (Willem) Kleijn

Victoria University of Wellington, bastiaan.kleijn@ecs.vuw.ac.nz

Follow this and additional works at: https://ro.uow.edu.au/eispapers

Part of the Engineering Commons, and the Science and Technology Studies Commons

Research Online is the open access institutional repository for the University of Wollongong. For further information contact the UOW Library: research-pubs@uow.edu.au 


\title{
Reproducing personal sound zones using a hybrid synthesis of dynamic and parametric loudspeakers
}

\author{
Abstract \\ This paper proposes a hybrid approach to personal sound zones utilising multizone soundfield \\ reproduction techniques and parametric loudspeakers. Crossover filters are designed, to switch between \\ reproduction methods, through analytical analysis of aliasing artifacts in multizone reproductions. By \\ realising the designed crossover filters, wideband acoustic contrast between zones is significantly \\ improved. The trade-off between acoustic contrast and the bandwidth of the reproduced soundfield is \\ investigated. Results show that by incorporating the proposed hybrid model the whole wideband \\ bandwidth is spatial-aliasing free with a mean acoustic contrast consistently above $54.2 \mathrm{~dB}$, an \\ improvement of up to $24.2 \mathrm{~dB}$ from a non-hybrid approach, with as few as 16 dynamic loudspeakers and \\ one parametric loudspeaker.
}

\section{Keywords}

hybrid, synthesis, dynamic, zones, parametric, reproducing, loudspeakers, sound, personal

Disciplines

Engineering | Science and Technology Studies

\section{Publication Details}

J. Donley, C. Ritz \& W. Bastiaan. Kleijn, "Reproducing personal sound zones using a hybrid synthesis of dynamic and parametric loudspeakers," in IEEE Asia-Pacific Signal and Information Processing Association Annual Summit and Conference (APSIPA), 2016, pp. 1-5. 


\title{
Reproducing Personal Sound Zones Using a Hybrid Synthesis of Dynamic and Parametric Loudspeakers
}

\author{
Jacob Donley*, Christian Ritz* and W. Bastiaan Kleijn ${ }^{\dagger}$ \\ * School of Electrical, Computer and Telecommunications Engineering, University of Wollongong, \\ Wollongong, NSW, 2522 Australia, E-mail: jrd089@uowmail.edu.au and critz@uow.edu.au \\ $\dagger$ School of Engineering and Computer Science, Victoria University of Wellington, \\ Wellington, 6140 New Zealand, E-mail: bastiaan.kleijn@ecs.vuw.ac.nz
}

\begin{abstract}
This paper proposes a hybrid approach to personal sound zones utilising multizone soundfield reproduction techniques and parametric loudspeakers. Crossover filters are designed, to switch between reproduction methods, through analytical analysis of aliasing artifacts in multizone reproductions. By realising the designed crossover filters, wideband acoustic contrast between zones is significantly improved. The trade-off between acoustic contrast and the bandwidth of the reproduced soundfield is investigated. Results show that by incorporating the proposed hybrid model the whole wideband bandwidth is spatial-aliasing free with a mean acoustic contrast consistently above 54.2dB, an improvement of up to $24.2 \mathrm{~dB}$ from a nonhybrid approach, with as few as $\mathbf{1 6}$ dynamic loudspeakers and one parametric loudspeaker.
\end{abstract}

\section{INTRODUCTION}

Personal sound environments, such as provided by multizone soundfield reproduction (MSR) [1] and parametric loudspeakers (PL) [2], are of interest in applications such as vehicle cabin entertainment/communication systems, cinema surround sound systems, multi-participant teleconferencing and personal audio in restaurant/cafés. As well as creating a target bright zone, it is sometimes also desired to create a second, quiet zone. In this case, it is important to ensure that the acoustic contrast (energy ratio) between zones is maximised whilst ensuring the error in the bright zone is minimised. However, research in the area has shown performance limitations related to audio leaking between zones, known as interzone audio interference, which limits the bandwidth of low error, high acoustic contrast, personalised audio.

The concept of personal sound from controlling multiple loudspeakers has been around since 1997 [3]. A method [4] was proposed later, in 2002, to maximise the ratio of energy between two regions which was termed Acoustic Contrast Control [1]. Afterwards, earlier multizone reproduction techniques made use of least-squares pressure matching [5] and cylindrical harmonic expansion [6]. Further research has made improvements in spatial reproduction accuracy by utilising planarity [7] and orthogonal basis planewaves [8].

Scenarios where a finite number of loudspeakers are used as secondary sources for soundfield reproduction, are limited to accurate reproduction below a (spatial aliasing) frequency [9]. A fundamental issue with MSR using discrete secondary sources is that the spatial aliasing induces so-called grating lobes which can interfere across zones [10]. Recent research [6], [8], [11] suggests a full circle array of $\approx 300$ loudspeakers are required to reproduce audio up to $8 \mathrm{kHz}$ with high acoustic contrast.

PLs, on the otherhand, are capable of providing high directivity at high frequencies [12] and were first theorised in 1963 [13]. PLs have gained interest due to their high directivity with a relatively small physical size which is comparable to dynamic (conventional) loudspeakers. Practical implementations have shown PLs can provide immersive spatial audio [14], [15], however, neither of the hybrid approaches use MSR with dynamic loudspeakers or consider spatial aliasing. When comparing PLs to MSR from dynamic loudspeakers, PLs lack directivity at low frequencies [12], contain higher Total Harmonic Distortion (THD) [2], [16] and can have potential health risks due to the high Sound Pressure Level (SPL) of the ultrasonic carrier frequency [2].

A hybrid system utilising the better aspects of both MSRs and PLs would allow for high acoustic contrast at low and high frequencies. Reproduction of speech soundfields [11], [17], [18] would require low carrier SPL in PLs due to the low energy of high frequency components in speech [19], thus reducing related health risks. Further, frequency dependent PL distortions are less of a problem at higher frequencies [16].

In this paper novel contributions are made through an analytical approach to a hybrid MSR and PL system with application to personal sound zones. A zone dependent crossover filter is designed to shift the loudspeaker signals between the MSR and PL in the frequency domain. A wideband acoustic contrast is presented for the hybrid system and the tradeoff between the acoustic contrast, crossover frequency and reproduced bandwidth is discussed.

Beginning this paper, in Section II, is an explanation of the MSR layout and soundfield reproduction aliasing. Section III gives a brief overview of the PL directivity model used in this work. In Section IV a hybrid method is formulated for MSR and PL reproduction of personal sound zones with results and discussion in Section V and conclusions in Section VI.

\section{Multizone SOUndField Reproduction (MSR)}

In this section a general MSR layout is described along with a description of a recent MSR technique. The aliasing which occurs from reproductions with spatial discretisation artifacts is also explained for later use in the hybrid model. 


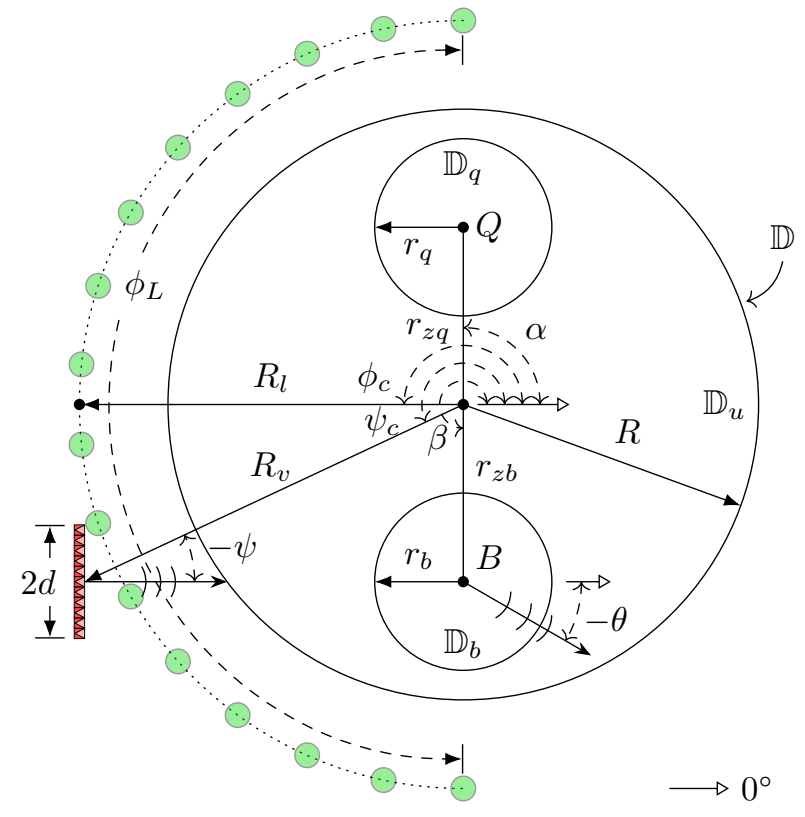

Fig. 1. MSR layout for a circular loudspeaker array (green) with a companion PL (red) for hybrid soundfield reproduction in $\mathbb{D}_{b}$.

In this work, the acoustical brightness contrast between two zones, $\mathbb{D}_{b}$ and $\mathbb{D}_{q}$, is defined as

$$
\zeta_{\mathcal{R}}(k)=\frac{\mathfrak{d}_{q} \int_{\mathbb{D}_{b}}\left|S_{\mathcal{R}}^{a}(\mathbf{x}, k)\right|^{2} d \mathbf{x}}{\mathfrak{d}_{b} \int_{\mathbb{D}_{q}}\left|S_{\mathcal{R}}^{a}(\mathbf{x}, k)\right|^{2} d \mathbf{x}},
$$

where $\mathfrak{d}_{b}$ and $\mathfrak{d}_{q}$ are the areas (sizes) of $\mathbb{D}_{b}$ and $\mathbb{D}_{q}$, respectively. The mean square error (MSE) between the desired soundfield, $S^{d}(\mathbf{x}, k)$, and the actual reproduced soundfield, $S_{\mathcal{R}}^{a}(\mathbf{x}, k)$, is [6], [20]

$$
\epsilon_{\mathcal{R}}(k)=\frac{\int_{\mathbb{D}_{b}}\left|S^{d}(\mathbf{x}, k)-S_{\mathcal{R}}^{a}(\mathbf{x}, k)\right|^{2} d \mathbf{x}}{\int_{\mathbb{D}_{b}}\left|S^{d}(\mathbf{x}, k)\right|^{2} d \mathbf{x}},
$$

which is used to measure reproduction accuracy. These measures can be used for any actual soundfield, $S_{\mathcal{R}}^{a}(\mathbf{x}, k)$, created with any reproduction technique, $\mathcal{R}$, such as MSR, PL or any combination thereof.

\section{A. MSR Layout}

The geometry of a generic MSR layout is depicted in Fig. 1 for a circular array with a companion PL. An MSR reproduction region, $\mathbb{D}$, of radius $R$ is shown and contains three sub-regions called the bright, quiet and unattended zone labelled $\mathbb{D}_{b}, \mathbb{D}_{q}$ and $\mathbb{D}_{u}=\mathbb{D} \backslash\left(\mathbb{D}_{b} \cup \mathbb{D}_{q}\right)$, respectively. The centre of $\mathbb{D}$ is the origin from which other geometrical locations are related. The centres of $\mathbb{D}_{b}$ and $\mathbb{D}_{q}$ have radius and angle pair polar coordinates $\left(r_{z b}, \beta\right)$ and $\left(r_{z q}, \alpha\right)$, respectively. The radius of $\mathbb{D}_{b}$ and $\mathbb{D}_{q}$ is $r_{b}$ and $r_{q}$, respectively, and the direction of the soundfield within the regions is $\theta$ and $\vartheta$, respectively. The MSR loudspeaker arc has a centre located at $\left(R_{l}, \phi_{c}\right)$ and subtends an angle of $\phi_{L}$. The directional PL has a centre located at $\left(R_{v}, \psi_{c}\right)$ and is directed at an angle of $\psi$ clockwise from the origin. In practice, the PL is a circular array of transducers, with effective radius $d$, protruding normal to the reproduction plane. In this work, the imaginary unit is $i=\sqrt{-1}$ and the Euclidean norm is denoted with $\|\cdot\|$. The wavenumber $k=2 \pi f / c$ is interchanged with frequency, $f$, under the assumption that the speed of sound, $c$, is constant.

\section{B. MSR Technique}

An infinite set of planewaves arriving from every angle is capable of entirely describing any arbitrary desired soundfield [21]. A soundfield fulfilling the wave equation, in this work, is denoted by the function $S(\mathbf{x}, k)$, where $\mathbf{x} \in \mathbb{D}$ is an arbitrary spatial sampling point. As shown in the orthogonal basis expansion approach [8], [20] to MSR, an additional spatial weighting function, $w(\mathbf{x})$, can be used to set relative importance between zones. The weighted MSR soundfield function used in this work can be written as

$$
S(\mathbf{x}, k)=\sum_{j} P_{j}(k) F_{j}(\mathbf{x}, k),
$$

where the orthogonal wavefields, $F_{j}(\mathbf{x}, k)$, have coefficients, $P_{j}(k)$, for a given weighting function and desired soundfield, $S^{d}(\mathbf{x}, k)$; and $j \in\{1, \ldots, J\}$ where $J$ is the number of basis planewaves [8].

The complex loudspeaker weights used to reproduce the soundfield in the (temporal) frequency domain are [22], [8]

$$
U_{l}(k)=\sum_{\bar{m}=-M}^{M} \frac{2 e^{i \bar{m} \phi_{l}} \Delta \phi_{s} \sum_{j}\left(P_{j}(k) i^{\bar{m}} e^{-i \bar{m} \rho_{j}}\right)}{i \pi H_{\bar{m}}^{(1)}\left(k R_{l}\right)},
$$

where $\rho_{j}=(j-1) \Delta \rho$ are the wavefield angles, $\Delta \rho=2 \pi / J$, $\phi_{l}$ is the angle of the $l^{\text {th }}$ dynamic loudspeaker from $0^{\circ}, \Delta \phi_{s}$ is the angular spacing of the loudspeakers, $H_{\nu}^{(1)}(\cdot)$ is a $\nu^{\text {th }}$-order Hankel function of the first kind and the modal truncation length [8] is

$$
M=\lceil k R\rceil .
$$

Here, $P_{j}$ is chosen to minimise the difference between the desired soundfield and the actual soundfield [8].

The actual soundfield from MSR is the result from superposition of all individual loudspeaker responses

$$
S_{\mathrm{MSR}}^{a}(\mathbf{x}, k)=G_{\mathrm{MSR}}(k) \sum_{l} U_{l}(k) T\left(\mathbf{x}, \mathbf{l}_{l}, k\right),
$$

where $G_{\mathrm{MSR}}(k)$ is introduced as an arbitrary weighting for hybrid soundfields (described later in IV-A), the loudspeaker's 2-D acoustic transfer function (ATF) is

$$
T\left(\mathbf{x}, \mathbf{l}_{l}, k\right)=\frac{i}{4} H_{0}^{(1)}\left(k\left\|\mathbf{x}-\mathbf{l}_{l}\right\|\right),
$$

and $\mathbf{l}_{l}$ is the position of the $l^{\text {th }}$ dynamic loudspeaker. Setting $G_{\mathrm{MSR}}(k)=1$ in (6) will render the multizone soundfield.

\section{Soundfield Reproduction Aliasing}

A fundamental issue with reproducing soundfields using a limited number of loudspeakers is spatial aliasing which gives rise to grating lobes which may impede the quiet zone at higher frequencies [10]. Due to this phenomenon, the bandwidth of reproducible soundfields with high acoustic contrast (which may be lost above the aliasing frequency) 
is reduced. For a part-circle array, the minimum number of dynamic loudspeakers to use before aliasing problems begin to occur is given by [6], [8]

$$
L \geq\left\lceil\frac{\phi_{L}(2 M+1)}{2 \pi}\right\rceil+1 .
$$

Substituting (5) into (8) and rearranging to find an approximation for upper frequency limit $k=k_{\mathrm{u}}$, gives

$$
k_{\mathrm{u}}=\frac{2 \pi(L-1)-\phi_{L}}{2 R^{\prime} \phi_{L}}
$$

where, instead of $R, R^{\prime}$ is used which is the radius of the smallest circle concentric with $\mathbb{D}$ encompassing all zones. The upper frequency from (9) agrees with [10] and is dependent on the number of loudspeakers, the reproduction radius and the angle subtending the loudspeaker arc.

\section{PARAMETRIC LOUDSPEAKER (PL)}

A few PL directivity models are reviewed in this section as well as common disadvantages of PLs. The disadvantages are discussed in regards to speech soundfields, further motivating the use of a hybrid model for such applications.

\section{A. Directivity Models}

The literature provides a handful of directivity models for PLs which are algorithmic approximations of the pressure at different angles. Earlier models include Westervelt's directivity (WD) [13] and product directivity (PD) [23], [24], though, these models do not accurately match measured directivity from a PL. Recently a convolutional directivity (CD) model, used in this work, was proposed [12], [25] utilising both WD and PD which has better correlation to measured directivity.

The actual soundfield reproduced by the PL, where the PL is located at $\mathbf{p}$, is defined in this work as

$$
S_{\mathrm{PL}}^{a}(\mathbf{x}, k)=G_{\mathrm{PL}}(k) E(\mathbf{x}, k) \mathcal{D}(\mathbf{x}, k) e^{i k\|\mathbf{x}-\mathbf{p}\|},
$$

where $G_{\mathrm{PL}}(k)$ is introduced as an arbitrary weighting for hybrid soundfields (described later in IV-A), $\mathcal{D}(\mathbf{x}, k)$ is the $\mathrm{CD}$ and the directivity coefficient is

$$
E(\mathbf{x}, k)=\tilde{\beta} k^{2} / 4 \pi \tilde{\alpha}_{s} \tilde{\rho}_{0}\|\mathbf{x}-\mathbf{p}\| c^{2},
$$

where $\tilde{\beta}$ is the coefficient of non-linearity, $\tilde{\alpha}_{s}$ is the sum of the absorption coefficients for both primary frequencies and $\tilde{\rho}_{0}$ is the density of the medium.

The CD is defined as the convolution between the PD and WD with the linear convolution operator, *, as [12], [25]

$$
\mathcal{D}(\mathbf{x}, k)=\left[\mathcal{D}_{\mathrm{G}}\left(\mathbf{x}, k_{\mathrm{c}}\right) \mathcal{D}_{\mathrm{G}}\left(\mathbf{x}, k_{\mathrm{c}}+k\right)\right] * \mathcal{D}_{\mathrm{W}}(\mathbf{x}, k),
$$

where $k_{\mathrm{c}}$ is the ultrasonic carrier frequency, $\mathcal{D}_{\mathrm{G}}(\mathbf{x}, \hat{k})$ is the Gaussian directivity [24]

$$
\mathcal{D}_{\mathrm{G}}(\mathbf{x}, \hat{k})=e^{\left(\frac{i}{2} d \hat{k} \tan \left(\rho_{\mathbf{x}}+\Psi\right)\right)^{2}},
$$

where $\rho_{\mathbf{x}}$ is the angle of vector $\mathbf{x}-\mathbf{p}$ from $0^{\circ}, \Psi=$ $\left(\psi+\psi_{c}-\pi\right)$ and WD is [25]

$$
\mathcal{D}_{\mathrm{W}}(\mathbf{x}, k)=\tilde{\alpha}_{s} / \sqrt{\tilde{\alpha}_{s}^{2}+k^{2} \tan ^{4}\left(\rho_{\mathbf{x}}+\Psi\right)} .
$$

The far-field PL soundfield can then be found using (11) and (12) in (10) with $G_{\mathrm{PL}}(k)=1$. However, as $k$ decreases $S_{\mathrm{PL}}^{a}(\mathbf{x}, k)$ approaches that of a point source and $\zeta_{\mathrm{PL}}(k)$ is consequently reduced. It is assumed in this work that the PL is designed such that grating lobes are negligible [26] and for different virtual source locations, multiple steerable PL arrays can be used [15], [26].

\section{B. PLs for Speech Soundfields}

While PLs have been studied extensively over the years there are still some drawbacks when it comes to reproducing loud and clear audible sound. Audible reproductions from PLs are known to require a large carrier SPL $(>110 \mathrm{~dB})$ for typical speech conversation levels of $\approx 60 \mathrm{dBA}$, which has potential inadvertent health risks [2]. Fortunately, for applications of speech soundfields, high SPLs from the PL are not necessary for high frequency $(\gtrsim 2 \mathrm{kHz})$ components of speech [19], further, harmonic distortions are lower above this frequency [16]. Taking into account the PL location so that the far-field demodulated audio [27] overlays $\mathbb{D}_{b}$ and under the assumption that high SPL from the PL is not required over $\mathbb{D}_{b}$, health risks from the PLs could be argued to be negligible.

\section{HYBRID MSR AND PL SYSTEM}

A hybrid MSR and PL system is presented in this section for use in personal sound zone applications. A crossover filter is designed to switch target audio in the (temporal) frequency domain to each of the constituent reproduction techniques.

\section{A. Crossover Filter Design}

Ideally the combination of low and high frequency acoustic contrast from $S_{\mathrm{MSR}}^{a}(\mathbf{x}, k)$ and $S_{\mathrm{PL}}^{a}(\mathbf{x}, k)$, respectively, is desired for personal sound zones. The weightings, $G_{\mathrm{MSR}}(k)$ and $G_{\mathrm{PL}}(k)$, are introduced in (6) and (10), respectively, in order to facilitate a hybrid soundfield, $S_{\mathcal{H}}^{a}(\mathbf{x}, k)$. When composing a hybrid soundfield it is natural to limit spectral distortion of the reproduction at the crossover frequency, for this, we propose the use of Linkwitz-Riley (LR) filters. Here, a low-pass $\hat{n}^{\text {th }}$ order LR filter with a roll-off of $6 \hat{n} \mathrm{~dB} /$ octave is a cascaded Butterworth filter

$$
H_{\mathrm{LR}}^{\urcorner}(k)=\mathcal{B}_{\frac{\hat{n}}{2}}\left(k / k_{\mathrm{u}}\right)^{-2},
$$

where $\mathcal{B}_{\frac{\hat{n}}{2}}$ are Butterworth polynomials of order $\frac{\hat{n}}{2}$ and $k_{\mathrm{u}}$ from (9) is suggested as the crossover frequency. The matching LR high-pass is

$$
H_{\mathrm{LR}}^{\ulcorner}(k)=\mathcal{B}_{\frac{\hat{n}}{2}}\left(k_{\mathrm{u}} / k\right)^{-2}
$$

and together the crossover magnitude response is

$$
\left|H_{\mathrm{LR}}^{\urcorner}(k)+H_{\mathrm{LR}}^{\ulcorner}(k)\right|=1 .
$$

In this work, the arbitrary MSR weighting is set to

$$
G_{\mathrm{MSR}}(k)=H_{\mathrm{LR}}^{\urcorner}(k),
$$

and the arbitrary PL weighting is

$$
G_{\mathrm{PL}}(k)=H_{\mathrm{LR}}^{\ulcorner}(k) \text {. }
$$


Using the new weights from (18) and (19) in (6) and (10), respectively, a hybrid, $\mathcal{H}$, soundfield is defined as the superposition of a set of reproduction methods, $\mathscr{R}$ (in this work the cardinality of $\mathscr{R}$ is 2 ), as

$$
S_{\mathcal{H}}^{a}(\mathbf{x}, k)=\sum_{\mathcal{R} \in \mathscr{R}} \frac{\mathfrak{d}_{b}\left|G_{\mathcal{R}}(k)\right| S_{\mathcal{R}}^{a}(\mathbf{x}, k)}{\int_{\mathbb{D}_{b}}\left|S_{\mathcal{R}}^{a}(\mathbf{x}, k)\right| d \mathbf{x}},
$$

where each component soundfield is normalised to the mean amplitude over $\mathbb{D}_{b} . \zeta_{\mathcal{R}}(k)$ and $\epsilon_{\mathcal{R}}(k)$ can be evaluated using $S_{\mathcal{H}}^{a}(\mathbf{x}, k)$ in place of $S_{\mathcal{R}}^{a}(\mathbf{x}, k)$ in (1) and (2), respectively.

\section{B. Loudspeaker Signals}

The time domain loudspeaker signals (unmodulated for a PL) are defined in general in this section for the reproduction of speech input signals, $y(n)$. The discrete Fourier transform of the $g^{\text {th }}$ overlapping windowed frame of $y(n)$ is $\tilde{Y}_{g}(k)$. The overlapping windowed frame of each loudspeaker signal is

$$
\begin{aligned}
\tilde{Q}_{\mathcal{R} l g}(k) & =\tilde{Y}_{g}(k) G_{\mathcal{R}}(k) U_{l}(k), \\
\tilde{q}_{\mathcal{R} l g}(n) & =\frac{1}{K} \sum_{m=0}^{K-1} \tilde{Q}_{\mathcal{R} l g}\left(k_{m} \hat{f}\right) e^{i c n k_{m}},
\end{aligned}
$$

where $k_{m} \triangleq 2 \pi m / c K$, the number of frequencies is $K$, the maximum frequency is $\hat{f}$ and each loudspeaker signal, $q_{\mathcal{R} l}(n)$, for a particular $\mathcal{R}$, is reconstructed by performing overlap-add reconstruction with the synthesis window on $\tilde{q}_{\mathcal{R} l g}(n)$. For the case where there is a single loudspeaker, $l=\{1\}$, for a given $\mathcal{R}$, such as for the PL in this work, $U_{l}(k)=1$ is used.

\section{REsults AND Discussion}

\section{A. Experimental Setup}

Simulations were carried out using the geometry shown in Fig. 1 with $r_{z b}=r_{z q}=0.6 \mathrm{~m}, r_{b}=r_{q}=0.3 \mathrm{~m}, R=1.0 \mathrm{~m}$ and $\alpha=\beta / 3=90^{\circ}$. The desired soundfield angle was $\theta=0^{\circ}$ and in this work $w(\mathbf{x})$ was set to one in $\mathbb{D}_{b}, 100$ in $\mathbb{D}_{q}$ and 0.05 in $\mathbb{D}_{u}$ based on [8], [11], [20]. The target soundfield in $\mathbb{D}_{b}$ was a virtual point source located at the centre of the PL and $\mathbb{D}_{q}$ was set to be quiet. The loudspeakers had $R_{l}=R_{v}=1.3 \mathrm{~m}$, $\phi_{L}=180^{\circ}, \phi_{c}=180^{\circ}$ and $\psi=\psi_{c}-180^{\circ}=27.5^{\circ}$. The speed of sound in air was $c=343 \mathrm{~m} \mathrm{~s}^{-1}$.

The PL was designed with $k_{\mathrm{c}}=2 \pi(40 \mathrm{kHz}) / c, \tilde{\beta}=1.2$, $\tilde{\alpha}_{s}=2.328 \mathrm{~m}^{-1}, \tilde{\rho}_{0}=1.225 \mathrm{~kg} \mathrm{~m}^{-3}$ and $d=6.18 \mathrm{~cm}$. In this work, it was assumed that the PL had ultrasonic transducer spacing less than $4.3 \mathrm{~cm}$ [26], thus avoiding spatial aliasing.

The LR filters used to reproduce $S_{\mathrm{MSR}}^{a}(\mathbf{x}, k)$ and $S_{\mathrm{PL}}^{a}(\mathbf{x}, k)$ had order $\hat{n}=12$. The number of MSR loudspeakers used was $L=\{16,24,32,134\}$ where $k_{\mathrm{u}}$ was found from (9). To compare with MSR, $L=134$ was chosen to reproduce the speech with no spatial aliasing. The hybrid reproduction method used $\mathscr{R}=\{\mathrm{MSR}, \mathrm{PL}\}$ to find $S_{\mathcal{H}}^{a}(\mathbf{x}, k)$ using (20).

\section{B. Wideband Spatial Error Reduction}

Figure 2 shows $\epsilon_{\mathrm{MSR}}(k), \epsilon_{\mathrm{PL}}(k)$ and $\epsilon_{\mathcal{H}}(k)$ computed from (2) in $(\mathrm{E})-(\mathrm{H})$ as dashed green, dashed red and solid blue lines, respectively. The crossover frequencies are the vertical dash-dot black lines. Comparing the proposed hybrid
TABLE I

WIDEBAND MEAN $\epsilon_{\mathcal{R}}$ AND $\zeta_{\mathcal{R}}$ COMPARISONS AS A FUNCTION OF THE NUMBER OF DYNAMIC LOUDSPEAKERS $(L)$ FOR ONE PL

\begin{tabular}{|c|ccc|ccc|}
\hline \multirow{2}{*}{$L$} & \multicolumn{3}{|c|}{$\epsilon_{\mathcal{R}}(\mathrm{dB})$} & \multicolumn{3}{c|}{$\zeta_{\mathcal{R}}(\mathrm{dB})$} \\
\cline { 2 - 7 } & MSR & PL & $\mathcal{H}$ & MSR & PL & $\mathcal{H}$ \\
\hline 16 & -27.2 & -40.7 & -32.5 & 30.0 & 40.4 & $\mathbf{5 4 . 2}$ \\
\hline 24 & -32.7 & -40.7 & -31.7 & 38.1 & 40.4 & $\mathbf{5 8 . 1}$ \\
\hline 32 & -33.7 & -40.7 & -31.6 & 43.5 & 40.4 & $\mathbf{6 0 . 3}$ \\
\hline \hline 134 & -36.4 & -40.7 & -35.6 & 79.6 & 40.4 & 79.3 \\
\hline
\end{tabular}

approach, it can be seen in Fig. 2 that $\epsilon_{\mathcal{H}}$ was on average similar to the aliasing free MSR. Table I confirms this by showing that, on average, $\epsilon_{\mathcal{H}}$ was slightly less than $\epsilon_{\mathrm{MSR}}$. While this was partly due to the low MSE of $\epsilon_{\mathrm{PL}}$ at lower frequencies, acoustic contrast was also reduced when using a PL at those lower frequencies as seen in Fig. 2 (A)-(D). The trade-off between MSE and acoustic contrast is shown in Table I where $\epsilon_{\mathcal{H}}$ reduces with $L$

\section{Wideband Acoustic Contrast Improvement}

Figure 2 shows $\zeta_{\mathrm{MSR}}(k), \zeta_{\mathrm{PL}}(k)$ and $\zeta_{\mathcal{H}}(k)$, computed from (1), in (A)-(D) as dashed green, dashed red and solid blue lines, respectively. The crossover frequencies are the vertical dash-dot black lines which clearly indicate the point where $\zeta_{\mathrm{MSR}}(k)$ begins to decrease due to spatial aliasing. Note that the multizone occlusion problem [1], [11] (should it occur) may be difficult to overcome with one PL, however, the MSR grating lobes interfere less over $\mathbb{D}_{q}$ during this phenomenon. Also shown in Fig. 2 is the limited bandwidth with high acoustic contrast when reducing $L$. The mean acoustic contrast over the wideband bandwidth for all reproduction techniques is given in Table I and the mean improvement using the hybrid method can be deduced. While the MSR mean acoustic contrast decreased significantly, from $79.6 \mathrm{~dB}$ to $30.0 \mathrm{~dB}$, due to spatial aliasing, the proposed hybrid method decreased to only $54.2 \mathrm{~dB}$. For all reduced loudspeaker cases the hybrid approach outperformed both MSR and PL methods. The maximum improvement was $24.2 \mathrm{~dB}$ when $L=16$ and for all cases the wideband acoustic contrast remained above $54.2 \mathrm{~dB}$, despite the fundamental spatial aliasing that occurred.

\section{CONCLUSIONS}

This paper has proposed a hybrid approach to personal sound zones, including speech soundfields. An analytical solution to the combination of MSR and PL soundfields is presented along with a solution to a robust crossover filter. The crossover filter is analytically derived from the geometry of the soundfield layout whilst taking into account spatial aliasing artifacts. Experimental results show that a significant improvement in acoustic contrast from non-hybrid MSR and PL soundfields of $24.2 \mathrm{~dB}$ and $19.9 \mathrm{~dB}$, respectively, is achievable. The proposed hybrid method also yields mean wideband acoustic contrast consistently above $54.2 \mathrm{~dB}$ with as few as 16 dynamic loudspeakers and a single PL. Some topics for future work are improving speech intelligibility contrast (SIC) and quality in private speech sound zones using hybrid techniques. 
Acoustic Contrast and Mean Squared Error for Reproduction Methods
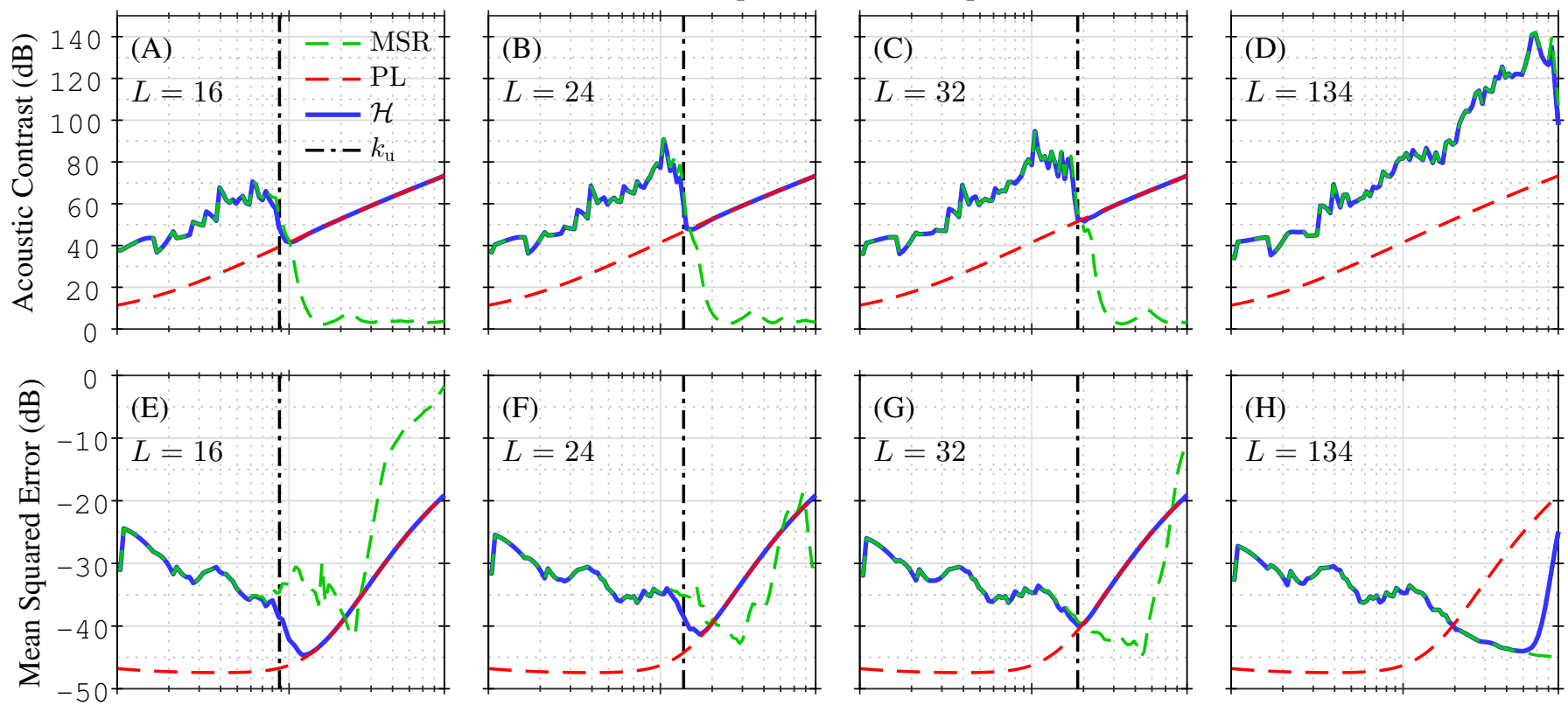

0.1

80.1

80.1

1

8

Fig. 2. Results are shown for three reproduction methods and four $L$. Acoustic contrast results $\left(\zeta_{\mathrm{MSR}}, \zeta_{\mathrm{PL}}\right.$ and $\left.\zeta_{\mathcal{H}}\right)$ are shown in (A)-(D). Mean squared error results $\left(\epsilon_{\mathrm{MSR}}, \epsilon_{\mathrm{PL}}\right.$ and $\left.\epsilon_{\mathcal{H}}\right)$ are shown in $(\mathrm{E})-(\mathrm{H})$. The case where $L=134$ is alias free up to $8 \mathrm{kHz}$.

\section{REFERENCES}

[1] T. Betlehem, W. Zhang, M. Poletti, and T. D. Abhayapala, "Personal Sound Zones: Delivering interface-free audio to multiple listeners," IEEE Signal Process. Mag., vol. 32, pp. 81-91, 2015.

[2] W.-S. Gan, J. Yang, and T. Kamakura, "A review of parametric acoustic array in air," Appl. Acoust., vol. 73, no. 12, pp. 1211-1219, Dec. 2012.

[3] W. F. Druyvesteyn and J. Garas, "Personal sound," J. Audio Eng. Soc., vol. 45, no. 9, pp. 685-701, 1997.

[4] J.-W. Choi and Y.-H. Kim, "Generation of an acoustically bright zone with an illuminated region using multiple sources," J. Acoust. Soc. Am., vol. 111, no. 4, pp. 1695-1700, 2002.

[5] M. Poletti, "An investigation of 2-D multizone surround sound systems," in Proc. 125th Conv. Audio Eng. Soc. Audio Eng. Soc., 2008, pp. 1-9.

[6] Y. J. Wu and T. D. Abhayapala, "Spatial multizone soundfield reproduction: Theory and design," IEEE Trans. Audio, Speech, Lang. Process., vol. 19, pp. 1711-1720, 2011

[7] P. Coleman, P. Jackson, M. Olik, and J. A. Pedersen, "Personal audio with a planar bright zone," J. Acoust. Soc. Am., vol. 136, pp. 1725-1735, 2014.

[8] W. Jin, W. B. Kleijn, and D. Virette, "Multizone soundfield reproduction using orthogonal basis expansion," in Int. Conf. on Acoust., Speech and Signal Process. (ICASSP). IEEE, 2013, pp. 311-315.

[9] S. Spors, H. Wierstorf, A. Raake, F. Melchior, M. Frank, and F. Zotter, "Spatial sound with loudspeakers and its perception: A review of the current state," Proc. IEEE, vol. 101, no. 9, pp. 1920-1938, 2013.

[10] F. Winter, J. Ahrens, and S. Spors, "On Analytic Methods for 2.5-D Local Sound Field Synthesis Using Circular Distributions of Secondary Sources," IEEE/ACM Trans. Audio, Speech, Lang. Process., vol. 24 no. 5, pp. 914-926, May 2016.

[11] J. Donley, C. Ritz, and W. B. Kleijn, "Improving speech privacy in personal sound zones," in Int. Conf. on Acoust., Speech and Signal Process. (ICASSP). IEEE, 2016, pp. 311-315.

[12] C. Shi, Y. Kajikawa, and W.-S. Gan, "An overview of directivity control methods of the parametric array loudspeaker," APSIPA Trans. Signal Inform. Process., pp. 1-30, Dec. 2014.

[13] P. J. Westervelt, "Parametric acoustic array," J. Acoust. Soc. Am., vol. 35, no. 4, pp. 535-537, 1963.

[14] Y. Sugibayashi, S. Kurimoto, D. Ikefuji, M. Morise, and T. Nishiura, "Three-dimensional acoustic sound field reproduction based on hybrid combination of multiple parametric loudspeakers and electrodynamic subwoofer," Appl. Acoust., vol. 73, no. 12, pp. 1282-1288, Dec. 2012.

[15] C. Shi, E.-L. Tan, and W.-S. Gan, "Hybrid immersive three-dimensional sound reproduction system with steerable parametric loudspeakers," in Proc. Meetings Acoust., vol. 19, 2013, pp. 1-6.

[16] C. Shi and Y. Kajikawa, "A comparative study of preprocessing methods in the parametric loudspeaker," in Asia-Pacific Signal \& Inform. Process. Assoc. Annu. Summit and Conf. (APSIPA ASC). IEEE, 2014, pp. 1-5.

[17] J. Donley and C. Ritz, "An efficient approach to dynamically weighted multizone wideband reproduction of speech soundfields," in China Summit \& Int. Conf. Signal and Inform. Process. (ChinaSIP). IEEE, 2015, pp. 60-64.

[18] _ - "Multizone reproduction of speech soundfields: A perceptually weighted approach," in Asia-Pacific Signal \& Inform. Process. Assoc. Annu. Summit and Conf. (APSIPA ASC). IEEE, 2015, pp. 342-345.

[19] Artificial voices. ITU-T Standard P.50, 1999.

[20] W. Jin and W. B. Kleijn, "Theory and design of multizone soundfield reproduction using sparse methods," IEEE/ACM Trans. Audio, Speech Lang. Process., vol. 23, pp. 2343-2355, 2015.

[21] E. G. Williams, Fourier Acoustics: Sound Radiation and Nearfield Acoustical Holography. Academic Press, 1999.

[22] Y. J. Wu and T. D. Abhayapala, "Theory and design of soundfield reproduction using continuous loudspeaker concept," IEEE Trans. Audio, Speech, Lang. Process., vol. 17, pp. 107-116, 2009.

[23] H. O. Berktay and D. J. Leahy, "Farfield performance of parametric transmitters," J. Acoust. Soc. Am., vol. 55, no. 3, pp. 539-546, 1974

[24] C. Shi and W.-S. Gan, "Product directivity models for parametric loudspeakers," J. Acoust. Soc. Am., vol. 131, no. 3, pp. 1938-1945, 2012.

[25] C. Shi and Y. Kajikawa, "A convolution model for computing the farfield directivity of a parametric loudspeaker array," J. Acoust. Soc. Am. vol. 137, no. 2, pp. 777-784, 2015.

[26] Chuang Shi and Woon-Seng Gan, "Grating lobe elimination in steerable parametric loudspeaker," IEEE Trans. Ultrason. Ferroelectr. Freq. Control, vol. 58, no. 2, pp. 437-450, 2011.

[27] F. Farias and W. Abdulla, "On Rayleigh distance and absorption length of parametric loudspeakers," in Asia-Pacific Signal \& Inform. Process. Assoc. Annu. Summit and Conf. (APSIPA ASC). IEEE, 2015, pp. 1262 1265. 